\title{
Research of Algorithms for Approaching and Docking Underwater Vehicle with Underwater Station
}

\author{
Vyacheslav Pshikhopov ${ }^{1}$, Boris Gurenko ${ }^{1, a}$, Maksim Beresnev ${ }^{1}$ \\ ${ }^{1}$ Southern Federal University, Rostov-on-Don, RUSSIA
}

\begin{abstract}
Authors consider problem of maintenance and service of underwater vehicles. Usually, underwater station or accompanying ship is required for such operations. Docking is one of the most difficult tasks on the vehicle path from the outer space to the servicing bay. Algorithms allowing docking were presented in the earlier paper, and in this paper authors prove their stability. Movement control is based upon the path regulator. The stability of the closedloop system according to Liapunov with the given control and limitations is proven. Equations, showing that vehicle will complete the positioning task with account to given limitations and staying stable are given. The criterion for switching movement and "positioning to point" algorithms is proposed. Achievement of the developed criterion was researched theoretically and in computer simulation. Experiments provide deviation of actual coordinates and velocity from the required ones and proved that achieving of criterion is enough to claim that system will be stable while performing algorithms with limitations for controls.
\end{abstract}

\section{Introduction}

Underwater vehicles (UV) are widely spread nowadays. Authors complete several projects concerning such vehicles, including development of mathematical model [1] and control system [2]. However, after the launch, periodical service and maintenance is crucial for the UVs. Special service equipment is carried onboard of the accompanying ship, submarine or even underwater station. In order to get there, vehicle needs to perform docking operation. To solve this task, three algorithms: general control algorithm, moving algorithm and vehicle position algorithm were presented in paper [3]. Now let us research proposed algorithms in terms of their stability and define a criterion for switching between them while approaching and docking.

\section{Research of movement algorithm}

To move UV to docking station area, it is necessary to create path regulator. Regulator should base on laws of position-path control, developed by V. Pshikhopov [2-5] but with regard to peculiarities of controlled object [6]. As it was shown in [6], mathematical model of UV in matrix form is:

$$
\begin{gathered}
\dot{\bar{Y}}=\Sigma(\bar{\Theta}, \bar{x})=\Sigma\left(\begin{array}{c}
\Sigma_{p}(\bar{\Theta}, \bar{x}) \\
\Sigma_{\Theta}(\bar{\Theta}, \bar{x})
\end{array}\right), \\
\widetilde{M} \dot{\bar{X}}=\left[\overline{F_{d}}(\bar{P}, \bar{V}, \bar{\omega})+\bar{F}_{u}(\bar{\delta})+\overline{F_{v}}\left(G, A_{p}, R_{g}\right)\right], \\
T_{c y} \frac{d \bar{\delta}}{d t}+\bar{\delta}=\overline{\Psi_{c y}}(\bar{\delta}, \bar{U}) .
\end{gathered}
$$

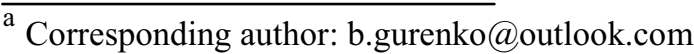

where $T_{c y}$ and $\overline{\Psi_{c y}}(\bar{\delta}, \bar{U})$ - diagonal matrix of time constants and vector of non-linear functions of right side of equations of actuation devices; $\delta$ - vector of control actions for UV, formed by actuation devices; $\bar{U}$ - control vector, formed by UV control system; $x-$ m-vector of internal coordinates; $M-(m \times m)$-matrix of UV mass and inertial parameters; $F_{\mathrm{u}}-\mathrm{m}$-vector of control forces and force moments; $F_{\mathrm{d}}-m$-vector of UV non-linear dynamics elements; $F_{\mathrm{v}}-$ m-vector of immeasurable external disturbances; $Y=(P, \Theta)^{T}-$ n-vector of position $P$ and orientation $\theta$ in body axis system; $n \leq 6 ; \sum(\Theta, \mathrm{x})-\mathrm{n}$ vector of kinematic links; $\sum_{p}(\Theta, \mathrm{x})$ - vector of linear velocities in body axis system; $\sum_{\Theta}(\Theta, \mathrm{x})$ - vector of angular velocities in body axis system

For the abovementioned model (1) the closed-loop system equation will be:

$$
\begin{gathered}
\Psi_{t r}+T_{1} \dot{\Psi}_{t r}+T_{2} \Psi=0 \\
\Psi_{c k}+T_{3} \Psi_{c k}=0 \\
\Psi_{t r}=A_{1} P+A_{2} \Theta+A_{3} \\
\Psi_{c k}=A_{4} V+A_{5},
\end{gathered}
$$

where

$$
\begin{aligned}
& A_{1}=\left[\begin{array}{lll}
0 & 0 & 0 \\
0 & k_{y} & 0
\end{array}\right], A_{2}=\left[\begin{array}{lll}
1 & 0 & 0 \\
0 & 1 & 0
\end{array}\right], A_{3}=\left[\begin{array}{c}
-\varphi^{*} \\
-k_{y} H_{3}
\end{array}\right] \\
& P=\left[\begin{array}{l}
x \\
y \\
z
\end{array}\right], \Theta=\left[\begin{array}{l}
\varphi \\
\psi \\
\gamma
\end{array}\right], A_{3}=\left[\begin{array}{c}
\phi_{3} \\
-k_{y} H_{3}
\end{array}\right] \\
& \varphi=\operatorname{tg}\left(z-z^{*} / x-x^{*}\right)-\operatorname{tg}\left(V_{z} / V_{x}\right)
\end{aligned}
$$


$\mathrm{A}_{4}=\left[\begin{array}{lll}1 & 0 & 0 \\ 0 & 1 & 0 \\ 0 & 0 & 1\end{array}\right], A_{5}=\left[\begin{array}{l}V_{x}-V_{x}^{*} \\ V_{y}-V_{y}^{*} \\ V_{z}-V_{z}^{*}\end{array}\right]$

$\left|V_{x}^{*} V_{y}^{*} V_{z}^{*}\right|^{T}$ - is a vector for setting required UV velocity. It should be noted that $V_{y}^{*}=V_{z}^{*}=0$ due to specificity of object [5].

Substitution of (3) into equation (2) with regard to UV mathematical model (1) gives the following expression for control:

$$
F_{u}=-M\left[\begin{array}{cc}
A_{4} & 0 \\
A_{1} A^{\prime} & A_{2} A_{w}
\end{array}\right]\left[\begin{array}{c}
T_{3} \Psi_{c r} \\
A_{1} \dot{A}^{\prime} V+A_{2} \dot{A}_{w} W+T_{2} \Psi_{t r}+T_{1} \dot{\Psi}_{t r}
\end{array}\right]
$$

Proposed approach to path regulator synthesis differs in separation of control channels thus simplifying regulator adjustment. Movement is decomposed in horizontal and vertical planes. This is usual logic [8] of mobile object control, so it simplifies implementation of the algorithm into existing systems. It stands to mention, that separation of control channels and movement decomposition results in loosing of proposed algorithms to position-path ones in case of complex movement where multicoupling influence is high.

\section{Definition of system stability}

On the basis of mathematical model $(2,3)$ in paper [1] the following control was developed:

$$
F_{u}-F_{d}-M R^{-1} A_{1}^{-1}\left(A_{1} \dot{R} X+T_{1} A_{1} R X+T_{2} \Psi\right),
$$

where $\Psi=\dot{\Psi_{t r}}+T_{!} \Psi_{t r}=A_{1} R X+T_{1}\left(A_{1} Y+A_{0}\right)$.

To show that system is instable, the expression (5) should be substituted to mathematical model (1).

$$
\left\{\begin{array}{c}
\dot{Y}=R X \\
\dot{X}=-R^{-1} A_{1}^{-1}\left[A_{1} \dot{R} X+\left(T_{1}+T_{2}\right) A_{1} R X+T_{2} T_{1}\left(A_{1} Y+A_{0}\right)\right]
\end{array}\right.
$$

Let us take Liapunov function in the following form:

$$
V(x)=\Psi^{T} \mathcal{Q} \Psi
$$

where $Q$ is a diagonal matrix of the corresponding size. Then, derivative of Liapunov function $V(x)$ will be:

$$
\begin{aligned}
\dot{V}(x)=2\left[A_{1} R X\right. & \left.+T_{1}\left(A_{1} Y+A_{0}\right)\right]^{T} \mathcal{Q}\left[A_{1} \dot{R} X+A_{1} R \dot{X}\right. \\
& \left.+T_{1} A_{1} R X\right] .
\end{aligned}
$$

Substitution of equation (9) to equation (11) will result in:

$$
\begin{gathered}
\dot{V}(x)=2\left[A_{1} R X+T_{1}\left(A_{1} Y+A_{0}\right)\right]^{T} \mathcal{Q}\left[-T_{2} A_{1} R X\right. \\
\left.-T_{1} T_{2}\left(A_{1} Y+A_{0}\right)\right] .
\end{gathered}
$$

Let us choose matrix such $Q$, that $Q T_{2}=C$, where $C$ is a diagonal matrix of the corresponding dimension. Then, derivative $\dot{V}(x)$ will be:

$$
\begin{aligned}
\dot{V}(x)=2\left[A_{1} R X+\right. & \left.T_{1}\left(A_{1} Y+A_{0}\right)\right]^{T} C\left[A_{1} R X+T_{1}\left(A_{1} Y\right.\right. \\
& \left.+A_{0}\right] .
\end{aligned}
$$

As $C$ is positive definite matrix, then $\dot{V}(x)$ function is negatively definite. According to Liapunov stability theorem, equilibrium state of our system is Liapunov stable.

To define stability if the system (1) and control (5) with account to addition of Barabashin-Krasovski, it is necessary to show that $X$ in Liapunov function is not the solution of our differential equation.

$$
\begin{gathered}
A_{1} R X+T_{1}\left(A_{1} Y+A_{0}\right)=0 \\
X=-R^{-1} A_{1}^{-1} T_{1}\left(A_{1} Y+A_{0}\right)
\end{gathered}
$$

Then $\dot{X}=-R^{-1} A_{1}^{-1} T_{1} A_{1} R X$.

Substitution of $X$ and $\dot{X}$ to equation (6) will result in:

$$
\begin{aligned}
\dot{X} \neq R^{-1} \dot{R} R^{-1} A_{1}^{-1} & T_{1}\left(A_{1} Y+A_{0}\right) \\
& +R^{-1} A_{1}^{-1} T_{1}^{2}\left(A_{1} Y+A_{0}\right) .
\end{aligned}
$$

Last equation proves that singular point of expression (10) is not a solution for the system.

\section{Research of system stability}

In real system, control of $\mathrm{u}$ values is limited due to physical and energetic limitation of actuation devices. Mathematic expression for the control is:

$$
u_{p}=\left\{\begin{array}{c}
u,\|u\|<u_{\max } \\
-u_{\max } \operatorname{sign}(u),\|u\| \geq u_{\max }
\end{array}\right.
$$

It's obvious, that with many control limitations, closed-loop system may become instable, that is inadmissible in real systems. As shown in papers [2, 3], such situation is pressing when solving positioning task. For example object is far from the positioning point, has excess velocity and cannot be stopped in the required time. To solve this problem it is proposed to develop a criterion, achievement of which would mean stability of the closed-loop system in performing positioning task with restrictions $u_{\max }$. Criterion will be developed basing on method, proposed in paper [7]. Taking into account that Liapunov function has (7) form and it's derivative (10) is negative and definite, i.e. system is asymptotically stable, then having bounded norm of initial conditions $\left\|\Psi_{0}\right\|$ vector $\Psi=R X+T_{1} \Psi_{t r}$, where $\Psi_{t r}=\left[\begin{array}{c}P-P^{*} \\ \Theta-\Theta^{*}\end{array}\right]$, the following inequation can be defined:

$$
\| \Psi\left(t, y_{0}, x_{0} \| \leq M<\infty, \lim _{t \rightarrow \infty} \Psi(t, y, x)=0,\right.
$$

In fact $\left\|\Psi_{0}\right\|$ determines a deviation of UV coordinates from the positioning point and velocity. Taking into account that Liapunov function and its derivative have forms (10) and (11), according to properties of quadratic forms it can be stated:

$$
\begin{gathered}
\lambda_{1}^{Q}\|\Psi\|^{2} \leq V(x)=\Psi^{T} \mathcal{Q} \Psi \leq \lambda_{n}^{Q}\|\Psi\|^{2} \\
\lambda_{n}^{Q}\|\Psi\|^{2} \leq \dot{V}(x)=-2 \Psi^{T} C \Psi \leq-\lambda_{1}^{C}\|\Psi\|^{2}
\end{gathered}
$$

Where $\lambda_{1}^{Q}, \lambda_{1}^{C}, \lambda_{n}^{Q}, \lambda_{n}^{C}$ - biggest and smallest eigenvalues of $Q$ and $C$ matrixes. In paper [8] it is shown that taking inequations (14) and having $\rho_{0}=\sqrt{\lambda_{n}^{Q} / \lambda_{1}^{2}}, v=\lambda_{1}^{C} / 2 \lambda_{n}^{C}$, the estimation of $\Psi$ vector norm can be:

$$
\|\Psi\| \leq \rho_{0}\left\|\Psi_{0}\right\| e^{-v t} .
$$

Comparing (13) and (15), $M$ could be equated to $\rho_{0}\left\|\Psi_{0}\right\|$. Let's modify control (5) to the following: $\left\|F_{u}\right\|=\left\|K_{u}+K_{\Psi} \Psi\right\|<\left\|F_{u}^{\max }\right\|$. With account to 
properties of vector norm $\|\Psi\| \leq \frac{\left\|F_{u}^{\max }\right\|-\left\|K_{u}\right\|}{\left\|K_{\Psi}\right\|}$ or with account to (11) $\rho_{0}\left\|\Psi_{0}\right\| \leq \frac{\left\|F_{u}^{\max }\right\|-\left\|K_{u}\right\|}{\|K \Psi\|}$, and thus:

$$
\left\|\Psi_{0}\right\| \leq \frac{\left\|F_{u}^{\max }\right\|-\left\|K_{u}\left(x_{0}, y_{0}\right)\right\|}{\left\|K_{\Psi}\left(x_{0}, y_{0}\right)\right\| \rho_{0}}
$$

Substitution of $F_{\mathrm{u}}=B_{\mathrm{u}}$ in (16) will give final form of Liapunov stability criterion for initial state norm.

$$
\left\|\Psi_{0}\right\| \leq \frac{\left\|B_{u}^{\max }\right\|-\left\|K_{u}\left(x_{0}, y_{0}\right)\right\|}{\left\|K_{\Psi}\left(x_{0}, y_{0}\right)\right\| \rho_{0}}
$$

Consequently, performing the inequation (17), system, described by the mathematical model (1), control algorithms (5) and limitations (11) will complete positioning task being stable.

\section{Computer simulation}

In order to research the achieving of criterion (17) when positioning UV in a single point, two experiments were conducted. In first the norm $\Psi 0$ was measured by deviation of coordinates, in second - deviation of UV velocity. Results are presented in Fig. 1 and 2.

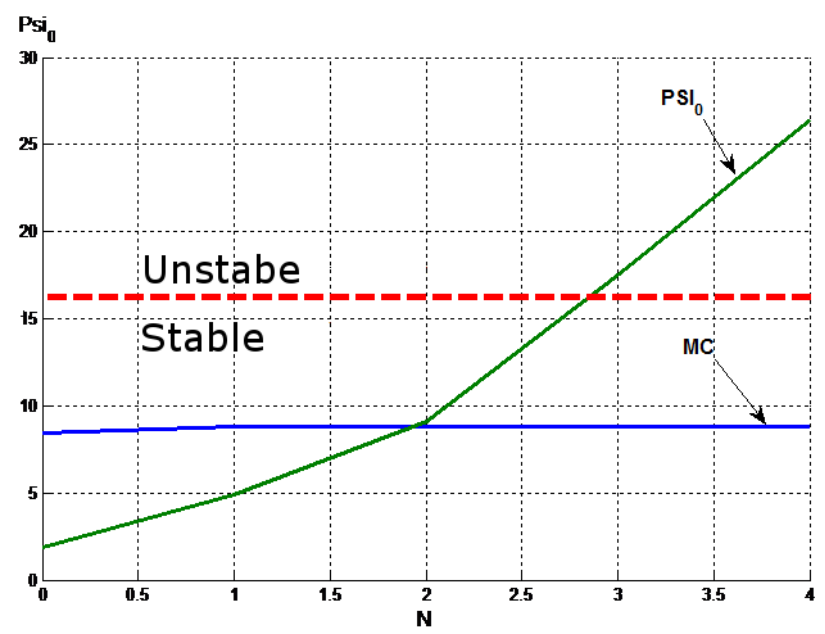

Figure 1. Change of stability of closed-loop system in variation of UV coordinates

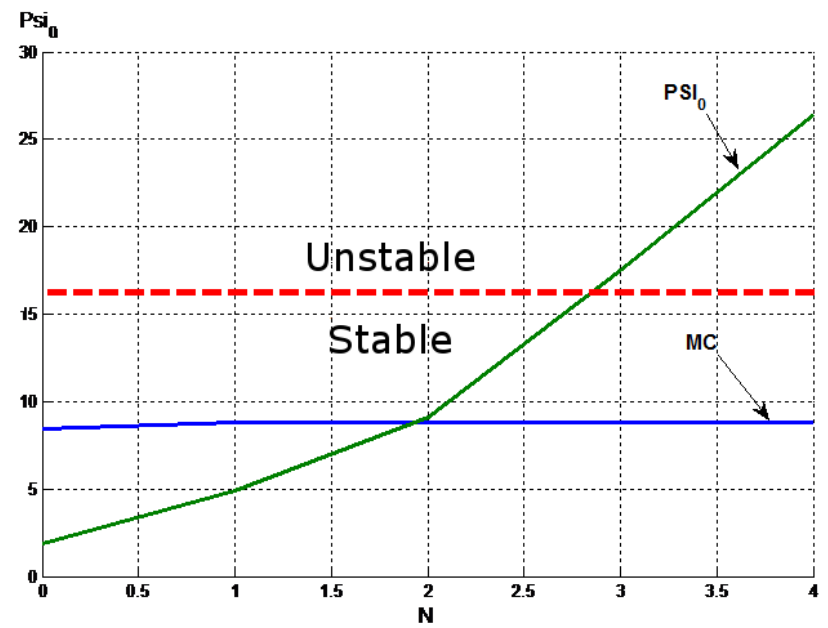

Figure 2. Change of stability of closed-loop system in variation of UV velocity.

\section{Conclusions}

The performed research proves the efficiency of the algorithms, developed earlier for underwater vehicle approaching and docking the underwater station. For the approaching part it is shown, that given system with stated control and limitations is stable in movement. Then, for docking part it is proven that vehicle is able to complete the positioning task. Problem of switching between movement and positioning algorithms was solved by development of switch criterion. It is based upon Liapunov stability criterion for initial state norm. Achieving of criterion allows system to be stable while performing algorithms with limitations for controls that was confirmed by computer simulation.

\section{Acknowledgements}

Research supported by Ministry of Education and Science of Russian Federation (research work No.114041540005) and Grant of the president of Russian Federation for state support of the Russian leading scientific school SSc-3437.2014.10.

\section{References}

1. B. Gurenko, Proc. of the Second Intl. Conf. on Advances In Mechanical and Robotics Engineering AMRE 2014. pp. 84-87, doi:10.15224/978-1-63248031-6-156 (2014)

2. V. Pshikhopov, Y. Chernukhin, A Fedotov, V. Guzik, M. Medvedev, B. Gurenko, etal. 4th International Workshop on Computer Science and EngineeringWinter, WCSE 2014, (2014)

3. B. Gurenko, and A. Beresnev, Submitted to ICMEA 2015, BangKok, Thailand (2015)

4. V. Pshikhopov, and M. Medvedev, IFAC World Congress, E 14, Part\# 1, pp. 10875-10880, doi: 10.3182/20110828-6-IT-1002.00006 (2011).

5. M. Medvedev, and V. Pshikhopov, Proceedings of 2010 IEEE Latin-American Conference on Communications (ANDERSON), pp.1-7, doi: 10.1109/ANDESCON.2010.5633481(2010)

6. V. Pshikhopov, M. Medvedev, M. Sirotenko, and V. Kostjukov, 9-th IFAC Symposium on Robot Control, pp. 26-31, doi: 10.3182/20090909-4-JP-2010.00007 (2009).

7. V. Pshikhopov, and A. Ali, Proceedings of IEEE International Conference on Mechatronics - ICM 2011, pp. $540 \quad-\quad 545, \quad$ doi: 10.1109/ICMECH.2011.5971345 (2011)

8. A. Gaiduk, K. Besklubova, A. Melnichenko, SFEDU times, E. 5, pp. 37-45 [in Russian].

9. A. Gaiduk, Facta universitatis-series: Automatic Control and Robotics, pp. 81-90, (2012). 